\title{
13
}

\section{Conservation Implications of Niche Conservatism and Evolution in Heterogeneous Environments}

\author{
Robert D. Holt and Richard Gomulkiewicz
}

\subsection{Introduction}

Species may, in principle, respond to environmental change in several different ways (Pease et al. 1989; Holt 1990; Chapters 10 and 11). Some species may track environmental states to which they are already well adapted and so shift in abundance and distribution. Other species may not evolve at all and so become extinct. Some species may evolve adaptively in ways that facilitate their persistence in changed environments. Yet other species may evolve in ways that hamper their long-term viability. A fundamental goal of the discipline of evolutionary conservation biology is to understand the factors that govern the relative likelihood of each of these outcomes.

Recognizing the importance of directional environmental change in driving extinctions in once-common species raises a profound puzzle. On the one hand, as ecologists we know that extinction risk emerges because directional environmental changes lead to lowered population abundances and/or restricted distributions; in effect, species are pushed outside their niches. On the other, as evolutionists we know that species often have abundant genetic variation, and so can adapt to novel circumstances. Conservation problems arise precisely because species do not adapt sufficiently to the new environments created by anthropogenic activity. In other words, conservation problems reflect a seeming failure of evolution by natural selection to adapt species to environmental change.

Such failures are examples of "niche conservatism". The history of life reveals examples of both niche conservatism (phylogenetic lineages that retain much the same ecological niche over substantial spans of evolutionary history) and niche evolution (Bradshaw 1991; Holt and Gaines 1992). Before proceeding any further, we should be clear about the meaning of "niche" (Schoener 1989). For a species with continuous, overlapping generations the intrinsic growth rate $r$ is its expected per capita birth rate minus its expected per capita death rate, at low densities. Succinctly, if a habitat results in $r>0$ for a given species it has conditions that are within that species' niche. By contrast, if $r<0$, the habitat has conditions outside the niche. (For discrete generations, if the environment is such that the per capita growth ratio per generation $R_{0}>1$, the habitat lies within the niche, but if $R_{0}<1$, it is outside.) In effect, the niche of a species is an abstract mapping of the most fundamental attribute of that species' population dynamics - its persistence versus its extinction - onto environmental states. A population of a species should persist 
(in the absence of stochastic fluctuations) if it experiences conditions within its niche, but go extinct if forced to live outside its niche.

Many conservation problems arise because environmental change forces a species' population outside that species' ecological niche. Evolution that influences extinction risk often involves niche evolution, such that species expand or shift their niches to incorporate novel environments. We do not downplay the role of other evolutionary processes in conservation (e.g., mutational meltdown in small populations, Lynch et al. 1995a; Chapter 9), but we do contend that an understanding of niche conservatism and evolution is integral to an evolutionarily informed conservation biology. In this chapter, we review theoretical studies which show that an absence of evolutionary responses in changed environments, which at first seems puzzling, actually makes sense when the demographic context of evolution is considered. Recent theoretical studies provide elements of a conceptual framework that allow niche conservatism to be understood, and possibly predicted. The basic idea is that population dynamics can, at times, constrain evolutionary dynamics. At other times, population dynamics facilitate evolutionary responses. This chapter provides an overview of these studies and highlights their implications for conservation.

Patterns of environmental change are complex in space and time. For conceptual clarity, we focus on simple situations with a step transition between two environmental states, or on spatial flows of individuals between two discrete habitats. We also briefly discuss evolution along smooth gradients in time and space. These different scenarios illustrate how demographic asymmetries can channel and constrain the evolution of local adaptation. We provide partial answers to two essential questions:

When does adaptive evolution mitigate extinction risk?

- Can we use our understanding of the dynamics of - and constraints on - adaptive evolution to guide practical conservation efforts?

\subsection{Adaptations to Temporal Environmental Change}

Consider a closed population, such as an insect species on an oceanic island or in an isolated habitat fragment. The population initially is found in a stable environment, at equilibrium within its niche. It then experiences an abrupt environmental change, and conditions shift to outside its niche (i.e., absolute fitness $R_{0}<1$ ). If the environment then stays constant, but the population does not evolve, extinction is certain (Figure 13.1a). Let us assume that the population has sufficient genetic variation to potentially persist in the novel environment. Selection increases average fitness, given the assumptions of Fisher's Fundamental Theorem (Fisher 1958; Burt 1995). However, as long as the average absolute fitness is less than 1, the population will continue to decline, though at a slowing rate. Eventually, mean fitness will exceed 1, and the population will start to increase. Such a species should display a characteristic U-shaped trajectory (Figure 13.1b).

Even so, this population may still experience a transient window of extinction risk. Given sufficient variation to adapt (i.e., evolve a positive growth rate) to 


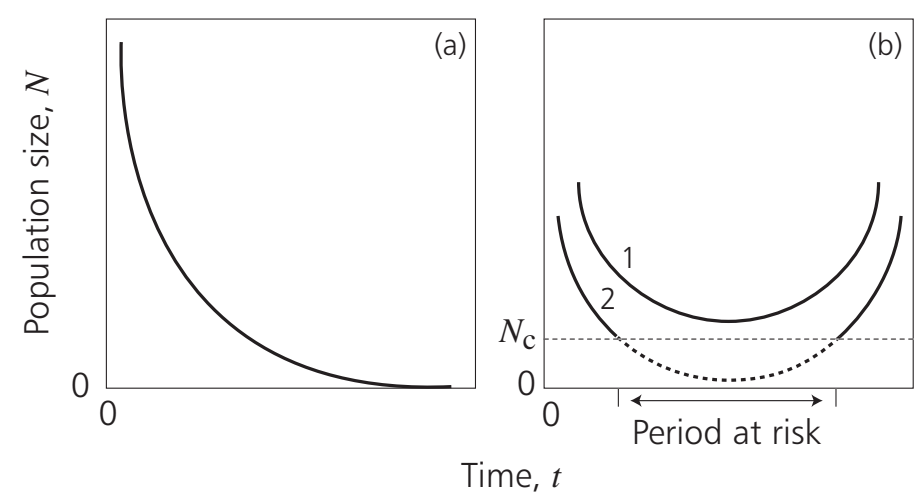

Figure 13.1 Population dynamics in a newly created sink habitat. (a) Without evolution: if evolutionary change is precluded (e.g., because relevant genetic variation is absent), population size declines toward extinction. (b) With evolution, if there is sufficient genetic variation, a population may adapt quickly (Trajectory 1), and so avoid exposure to the critically low sizes that are associated with high extinction risk (i.e., $N_{t}>N_{\mathrm{c}}$ for all $t$ ). However, if the population adapts more slowly (Trajectory 2), it spends a period at low abundances, and thus incurs a high risk of extinction (i.e., $N_{t}<N_{\mathrm{c}}$ for some span of time $t$ ). If by chance extinction is avoided and adaptation continues, the population may eventually rebound.

abrupt environmental change, in a deterministic world a population should eventually bounce back from low numbers. But when numbers become too low, even well-adapted populations may face extinction from demographic stochasticity. The dotted line in Figure 13.1b marks a critical population size $N_{\mathrm{c}}$ below which we assume demographic stochasticity to become a severe problem. For Trajectory 1 in Figure $13.1 \mathrm{~b}$ the population starts at a high density and evolves a positive growth rate sufficiently fast to rebound before ever entering the "danger zone" of low numbers. This results in a process of "evolutionary rescue". By contrast, for Trajectory 2 the initial density is lower and the population evolves slowly, so it experiences a period of extinction risk. Figure 13.1b illustrates a race between an evolutionary process (improved adaptation to the novel environment, increasing mean fitness), and an ecological process (declining numbers, as long as mean fitness is less than 1). In Box 13.1 we present an analytic model that explicitly combines evolution in a quantitative character and population dynamics and results in U-shaped trajectories in numbers.

Given a critical population size $N_{\mathrm{c}}$ below which extinction is probable, we can use Equation $(\mathrm{g})$ in Box 13.1 to determine whether or not a population trajectory includes periods of risk (i.e., $N_{t}<N_{\mathrm{c}}$ ). Figure 13.2 summarizes whether or not a population experiences such extinction risk as a function of its initial density, the degree of initial maladaptation in the novel environment, and the heritability of the trait that undergoes selection. The basic messages are as follows:

- Populations that are initially rare are highly vulnerable to even moderate environmental change;

- Even large populations are vulnerable to strong environmental change;

Evolutionary rescue is facilitated by increased genetic variability. 


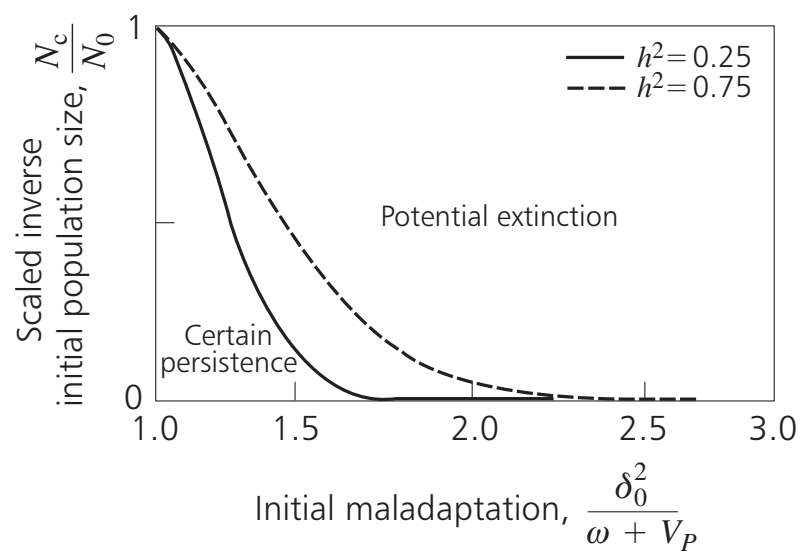

Figure 13.2 Combinations of initial population size and initial maladaptation that lead to high extinction risk. The vertical axis is an inverse measure of the initial abundance (scaled relative to a critical low size $N_{\mathrm{c}}$ ). The measure of initial maladaptation used is $\delta_{0}^{2} /\left(\omega+V_{P}\right)$, where $\delta_{0}$ is the distance between the optimal phenotype in the new sink environment and the initial mean phenotype of the population, $\omega$ is an inverse measure of the strength of selection, and $V_{P}$ is the phenotypic variance for the character being selected. The quantitative genetic model presented in Box 13.1 is used to derive curves that separate those situations that lead to population sizes always above $N_{\mathrm{c}}$ (lower left region) from those in which population sizes fall below $N_{\mathrm{c}}$ for a period of time of high extinction risk (upper right region). Results are shown for two values of heritability, $h^{2}=0.25$ and 0.75 . Source: Gomulkiewicz and Holt (1995).

The analytically tractable model described in Box 13.1 helps to clarify when evolution may rescue populations from extinctions. However, the model does not describe extinction directly, for it assumes continuous and deterministically variable densities, whereas individuals are discrete and numbers change stochastically. A direct assessment of extinction requires the use of models in which these features are respected, which implies counting individuals and alleles. Individual births, deaths, mating events, and mutations are all fundamentally stochastic processes (see Chapters 2 and 3). Analytical treatment of stochastic models that couple demographic and genetic dynamics for finite populations is a challenging task, even for simple one-locus situations (see Gomulkiewicz et al. 1999). An alternative approach that gives much insight is to use individual-based numerical simulations. We present results from such simulations, in which we track each individual and gene (locus by locus), and directly assess the probability of extinction by repeated simulations [extinction occurs when a population declines to zero abundance, and the probability of extinction in a given environment is the relative frequency of extinctions over a fixed time period for a large number of simulation runs; see Holt et al., unpublished, for technical details of the simulation protocol, which follows that of Bürger and Lynch (1995)]. Box 13.2 describes the genetic, life-history, and ecological assumptions of these individual-based simulations. The two approaches - analytic treatment and individual-based numerical simulations - lead to mutually reinforcing insights about the potential for evolutionary rescue. 
Box 13.1 Modeling adaptation and persistence after environmental change

Here an analytically tractable model to assess the propensity for evolutionary rescue is introduced. More details about the model can be found in Gomulkiewicz and Holt 1995 (see also Holt and Gomulkiewicz 1997b).

For a population with discrete generations, the finite rate of increase in population size $N_{t}$ in generation $t$ is just the mean fitness $\bar{W}_{t}, N_{t+1}=\bar{W}_{t} N_{t}$. We assume that a population is initially at an evolutionary equilibrium in a closed environment, and then experiences an abrupt change in environmental conditions. The average individual in the population is maladapted to the novel environment, so much so that in generation 0 after the environmental change $\bar{W}_{0}<1$. (We assume the density is low enough at this time to ignore density dependence.) If there is no evolutionary response, extinction results.

To couple population dynamics to evolution, we assume fitness depends upon a single trait $z$ with polygenic autosomal inheritance, such that

$$
W(z)=W_{0} \exp \left(-\frac{1}{2} z^{2} / \omega\right) .
$$

For convenience, we assume that the optimal phenotype in the new environment is at $z=0$, and that the initial mean phenotype in the new environment is $\delta_{0}$. The quantity $\omega$ is an inverse measure of the fitness cost of deviations from the optimum. Quantitative traits are often normally distributed, measured on an appropriate scale. We thus assume that the phenotypic distribution $p_{t}$ in generation $t$ can be described by

$$
p_{t}(z)=\left(2 \pi V_{P}\right)^{-1 / 2} \exp \left(-\frac{1}{2}\left(z-\delta_{t}\right)^{2} / V_{P}\right) .
$$

Here $V_{P}$ is the phenotypic variance, and $\delta_{t}$ is the distance of the mean phenotype in generation $t$ from the new optimum at $z=0$. The mean fitness in generation $t$ is

$$
\bar{W}_{t}=\int W(z) p_{t}(z) d z=\hat{W} \exp \left(-\frac{1}{2} \delta_{t}^{2} /\left(V_{P}+\omega\right)\right),
$$

where $\hat{W}=W_{0} \sqrt{\omega /\left(V_{P}+\omega\right)}$ is the growth rate attained when the mean phenotype is optimized.

In standard quantitative-genetic models of selection (Falconer 1989), the mean phenotype of a trait experiencing directional selection changes in accordance with

$$
\Delta \delta_{t}=\delta_{t+1}-\delta_{t}=h^{2} S,
$$

where $h^{2}$ is the trait's heritability (a measure of faithfulness in genetic transmission of trait values across generations), and $S$ is the selection differential (the difference in mean phenotype between individuals selected to be parents of the next generation and the mean phenotype of the current generation). For our model, we obtain

$$
S=\int z\left[W(z) / \bar{W}_{t}\right] p_{t}(z) d z-\delta_{t}=-\frac{\delta_{t} V_{P}}{V_{P}+\omega},
$$

and hence 
Box 13.1 continued

$$
\delta_{t+1}=\delta_{t}+\Delta \delta_{t}=\frac{\omega+\left(1-h^{2}\right) V_{P}}{V_{P}+\omega} \delta_{t}=k \delta_{t} .
$$

The quantity $k$ gauges evolutionary inertia; if $h^{2}$ is near zero, $k \approx 1$, and evolution is slow. As time passes, the mean phenotype approaches the local optimum, and the growth rate increases. Some algebra then shows the dynamics of population size to be described by

$$
N_{t}=N_{0} \hat{W}^{t} \exp \left(-\frac{\delta_{0}^{2}\left(1-k^{2 t}\right)}{2\left(V_{P}+\omega\right)\left(1-k^{2}\right)}\right) .
$$

This expression gives U-shaped population trajectories, comparable to those in Figure 13.1b.

Figure 13.3 shows the probability of extinction over 1000 generations (averaged over 400 independent simulation runs, except for the $b=3$ curve in Figure 13.3d, which is averaged over 1600 runs), as a function of the magnitude of the abrupt change in the phenotypic optimum caused by environmental change, and as influenced by genetic, life-history, and ecological parameters. Figure 13.3a depicts the influence of initial population size (carrying capacity) on extinction. Populations that are initially small (low $K$ ) or highly maladapted (large change in the optimum phenotype) have a high risk of extinction after an abrupt environmental change. Moreover, a large population does not, by itself, provide insurance against extinction if the degree of initial maladaptation is high. Changes in the number of loci that govern genetic variation in the trait have only a minor effect on the probability of extinction (although single-loci variation seems to hamper persistence with respect to polygenic variation, see Figure 13.3b). Populations with a higher mutational input of variation survive longer in the changed environment (Figure 13.3c). Species with high fecundities can tolerate more severe changes in the environment (Figure 13.3d). These conclusions qualitatively match those drawn from the extinction model described in Box 13.1 and summarized in Figure 13.2.

If the opportunity for niche evolution occurs primarily through sporadic colonization of novel environments outside a species' current niche, or because a species' entire population uniformly experiences severe, rapid environmental degradation, niche shifts would rarely save species from extinction. This is because populations that experience strong selection on niche characters are precisely those that face a severe risk of extinction. Species that are initially rare or have low fecundity may be particularly sensitive to environmental change. The degree to which such species persist will reflect their ability to disperse, tracking across space the shifting locations of environments to which they are already adapted. 
Box 13.2 An individual-based model for analyzing niche evolution

The individual-based simulation model introduced here allows us to examine both closed populations after abrupt environmental change and spatially discrete scenarios in which stable sources are coupled with migration to sink habitats (Holt et al., unpublished).

The model is based on assumptions made by Bürger and Lynch (1995), who studied adaptation to a continually changing environment for a single polygenic character:

Genetic assumptions: (a) additive effects of loci, without dominance or epistasis (each allele contributes a fixed amount to the phenotypic value, and an individual's phenotype is the sum of this quantity over all loci, plus a random term); (b) mutational input maintains variation, following the "continuum-ofalleles" model, in which mutational effects are drawn from a normal distribution; (c) free recombination; and (d) in the spatial model, the source population is in mutation-selection-drift balance.

- Life-history assumptions: (a) discrete generations; (b) dioecious, hermaphroditic, monogamous, and random mating.

- Ecological assumptions: (a) in the spatial model, a constant number of immigrants per generation; (b) "ceiling" density dependence (i.e., population growth is density independent below the carrying capacity, at which growth stops abruptly); (c) constant fecundity per mated pair; (d) offspring survival probability is a Gaussian function of phenotype.

A census is made of the adults to determine the population size $N_{t}$ in generation $t$. After the census, in the spatial model there is immigration at a per generation rate $I$, followed by random mating. The mating population is limited by a ceiling: if there are more than $K$ adults, $K$ individuals are sampled, without replacement from the pool, and are randomly assigned to mating pairs. Individuals produce gametes with free recombination among $n$ loci. Mutation occurs on gametes, with a stochastic mutational input $n \mu$ per genome (distributed randomly over all loci). Each mated pair produces $b$ offspring, which survive to adulthood with probability $p_{i}(z)=\exp \left(-\frac{1}{2}\left(z-\hat{z}_{i}\right)^{2} / \omega^{2}\right)$, where $z$ is the realized phenotype of a given individual, $\hat{z}_{i}$ is the optimum phenotype in habitat $i(1=\operatorname{sink}, 2=$ source $)$, and $\omega^{2}$ is inversely proportional to the strength of stabilizing selection. Survival to adulthood is the life-history stage at which selection occurs. If the realized phenotype is too far from the optimum, the mean fitness is below 1, and the population tends to decline. Individuals that survive early mortality are adults at the next census, $N_{t+1}$. The population is assumed to be at carrying capacity $K$ initially and at selectionmutation-drift equilibrium in the ancestral environment.

In this individual-based model, stochasticity enters at several stages:

Mutation is stochastic;

Gametic combinations and immigrants (in the spatial model) have multilocus allelic combinations that vary through random sampling; 


\section{Box 13.2 continued}

- Finally, survival is probabilistic, which leads to both genetic drift and chance fluctuations in population size.

To assess the summary statistics (e.g., the probability of extinction, or adaptation, over a given number of generations), many simulations are run, starting with identical initial conditions, and the relative frequencies of various outcomes are assessed.

One virtue of individual-based simulation models is that they enable comparison of the impact of various assumptions about the environment, and biology, for scenarios that are very difficult to tackle with analytical models. For example, does linkage among loci facilitate, or hamper, local adaptation? What is the effect of overlapping generations on niche evolution?

\subsection{Adaptations in Population Sources and Sinks}

A frequent scenario in real-world conservation crises is for a species to experience environmental degradation in only part of its geographic range. For instance, the localized dumping of toxins or invasion by exotic species could affect a species in certain areas, but not in others. Over time, many spatial patterns of habitat alteration could occur. For instance, degradation caused by the diffusion of a toxin could generate a smooth gradient in habitat quality, emanating from a point toxin source. By contrast, land clearance could lead to the abrupt juxtaposition of discrete habitat types in a complex mosaic.

A simple, but instructive, scenario is to assume that after habitat degradation there remains a discrete "source" habitat, in which the environment is unaltered and the species can persist at its ancestral evolutionary equilibrium. The degraded part of the species' range is represented by a discrete "sink" habitat, in which the conditions are so hostile that the species would go extinct but for recurrent immigration from the source. As used here, a "source" habitat is one in which births exceed deaths at low densities, so populations are expected to persist. By contrast, in a "sink" habitat there are fewer births than deaths, so populations decline to extinction in the absence of immigration (Pulliam 1996). Demographic sinks can also occur if immigration pushes the population size above the local carrying capacity, which gives rise to "pseudosinks" as discussed by Watkinson and Sutherland (1995).

Niche conservatism occurs if adaptive evolution to the sink habitat does not take place, even though the species is exposed to such evolution via immigration from the source. Should sink habitats concern conservationists? Sometimes the answer is surely "yes". Adaptation to poor environments may enhance the survival prospects for an entire species; indeed, it may be essential if a species' original habitat shrinks to pathetic fragments of a formerly extensive range. Models of adaptive evolution in the context of source-sink dynamics give insight into the potential for such adaptive responses. 


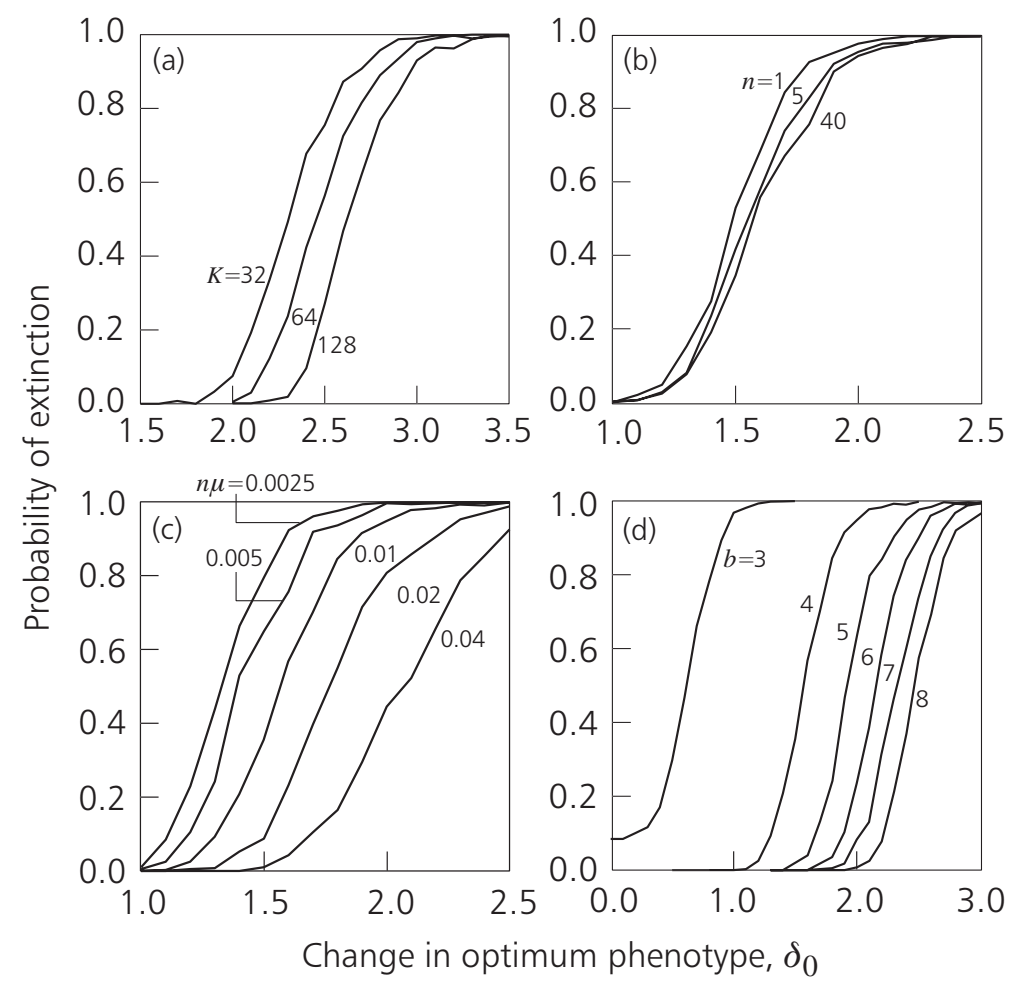

Figure 13.3 Probability of extinction over a span of 1000 generations for an individualbased model of a closed population that experiences an abrupt environmental transition. The initial genetic variability results from mutation-selection-drift balance for an ancestral population in a constant, favorable environment. When a mutation occurs in the simulations, a normally distributed random number with mean 0 and variance $V$ is added to the current allelic value. (a) Effect of the initial density, which equals the carrying capacity $K$ in all examples. Other parameters: $b=8, n=10, n \mu=0.01, \omega^{2}=1, V=0.05$. (b) Effect of numbers of loci $n$. Other parameters as in (a) with $K=64$, except for $b=4$. (c) Effect of mutational input of variation $n \mu$. Other parameters as in (b) with $n=10$. (d) Effect of fecundity $b$. Other parameters as in (b) with $n=10$.

To understand the interplay of migration and selection that determines local adaptation is a classic problem in population genetics (Hedrick 2000; see also Chapter 12), stemming back to J.B.S. Haldane (1930). However, this literature traditionally ignored the demographic context within which gene flow and selection occur. A very simple model that explicitly illustrates the importance of demography (Holt and Gomulkiewicz 1997a, 1997b) rests on the assumption that an asexual population with discrete generations is situated on a habitat patch. This population receives recurrent immigrants at a constant rate $I$ (number of immigrants per generation), all fixed for an allele $\mathrm{A}_{2}$. The absolute fitness of the immigrants is $W_{2}<1$. With these assumptions, the number of individuals on the patch follows the recursion $N^{\prime}=N W_{2}+I$, which implies the population equilibrates at $N^{*}=I /\left(1-W_{2}\right)$. Now assume a novel mutant allele $\mathrm{A}_{1}$ arises with higher fitness in the local environment, $W_{1}>W_{2}$. Can this allele spread by natural selection? In each generation, the relative frequency of the allele increases because 
of local selection, but it is also reduced because of the dilution by immigration of individuals that carry the less-fit allele. The recursion that describes the net effect of these two processes on the frequency $p$ of allele $\mathrm{A}_{1}$ is $p^{\prime}=(1-m)\left(W_{1} / \bar{W}\right) p$, where $\bar{W}=p W_{1}+(1-p) W_{2}$ is the mean fitness. The quantity $m$ measures gene flow, which is the percentage of the island population that comprises immigrants; after immigration, $m=I / N^{\prime}$. When the novel mutant is very rare, the population consists primarily of the less-fit immigrants, so the total population size is approximately $N^{*}$. After substitution, we find that the recursion for the frequency of the rare fitter allele becomes $p^{\prime}=W_{1} p$. The condition for the fitter allele to increase in frequency is that its absolute fitness exceeds 1 , irrespective of the fitness of the less-fit allele or of the rate of immigration. The conclusions from this very simple model hold much more broadly (Gomulkiewicz et al. 1999). Box 13.3 describes a one-locus model for a "black-hole" sink: a habitat that receives immigrants, but does not export emigrants back to the source (which is assumed to have a reproductive surplus providing the flux of immigrants).

These genetic models lead to interesting conclusions in terms of conservation. When considering the fate of mutant alleles in a sink habitat, absolute - not relative - fitness is key to adaptive evolution. The "effect" of a mutation is measured relative to an ancestral condition (here, fitness of the immigrant). In a "harsh" sink environment, an immigrant has a fitness well below 1. In such environments, only mutants that have a large effect on fitness can be retained and swept to higher frequencies by selection. By contrast, in a mild sink the absolute fitness of an immigrant is less than, but close to, 1 . In such an environment, mutants of small effect may be selected. If the rate of adaptation is limited primarily by the appearance of appropriate mutations, and mutants of small effect appear more frequently than do mutants of large effect (e.g., Orr 1998), then adaptation to a mild sink occurs more rapidly than does adaptation to a harsh sink. In effect, niche conservatism (the absence of adaptive niche evolution) is more likely given sharp contrasts in fitness between source and sink habitats.

What about immigration? If fitness is density independent in the sink environment, then because the rate of immigration $I$ drops out of the recursion, the magnitude of immigration of maladapted genotypes does not directly influence the initial spread of the locally favored allele. If, instead, fitness is density dependent, declining with population size $N$, immigration can directly hamper adaptation, because increasing immigration increases local abundances, and thus depresses local fitness. Moreover, even if fitness is initially density independent, once a locally favored allele has arisen and spread, the population size will rise, and eventually the absolute fitness must become density dependent. At the new demographic equilibrium, recurrent immigration can lower the frequency of the fitter allele because of such density dependence. Moreover, in a diploid model, mating between immigrants and residents further lowers local fitness, because of the continued generation of less-fit heterozygotes. Thus immigration can hamper the degree of adaptation to the sink environment for both ecological and genetic reasons. 
Box 13.3 A diploid, one-locus model for adaptation in a "black-hole" sink

To gain insight into how genetic structure affects adaptation in a "black-hole" sink (a habitat that receives immigrants, but does not export emigrants), we assume that fitness is governed by variation at a diploid locus with two alleles, $A_{1}$ and $A_{2}$. While allele $A_{1}$ is assumed to be favored in the sink, all immigrants are fixed for $\mathrm{A}_{2}$. The population size in the sink is given by $N$, and $p$ is the frequency of allele $\mathrm{A}_{1}$. In each generation, $I$ adults immigrate into the sink habitat (after selection, but before reproduction); subsequently, random mating occurs and a census is taken of the population. The viability of an individual with genotype $\mathrm{A}_{i} \mathrm{~A}_{j}$ is $v_{i, j}$, and all individuals have the same fecundity $b$. The fitness of genotype $\mathrm{A}_{i} \mathrm{~A}_{j}$ is thus $W_{i, j}=b v_{i, j}$. As the habitat is a sink for the immigrant genotype type $\mathrm{A}_{2} \mathrm{~A}_{2}$, $W_{22}<1$. We are interested in assessing the fate of the locally more favorable allele $\mathrm{A}_{1}$; hence, we assume $W_{12}>W_{22}$.

With these assumptions, the number of breeding adults (after viability selection and immigration) is

$$
N_{b}=v_{11} N p^{2}+v_{12} N 2 p(1-p)+v_{22} N(1-p)^{2}+I .
$$

The first three terms describe the Hardy-Weinberg distribution of genotypes, as modified by differential mortality; the total population consists of survivors of selection plus immigrants (fourth term).

After reproduction, the density of newborns is

$$
N^{\prime}=b N_{b}=N \bar{W}+b I,
$$

where $\bar{W}=W_{11} p^{2}+W_{12} 2 p(1-p)+W_{22}(1-p)^{2}$ is the mean fitness. The frequency $p^{\prime}$ of the $\mathrm{A}_{1}$ allele among newborns equals the frequency of $\mathrm{A}_{1}$ in the breeding parents; thus $p^{\prime}=$ (number of $\mathrm{A}_{1}$ alleles in parents) $/\left(N_{b}\right)$ and therefore

$$
p^{\prime}=\frac{1}{2 N_{b}}\left[2 v_{11} N p^{2}+v_{12} N 2 p(1-p)\right]
$$

Multiplying both the numerator and denominator of this expression by $b$ and using Equation (b) gives

$$
p^{\prime}=\frac{N}{N^{\prime}} \bar{W}_{1} p
$$

where $\bar{W}_{1}=p W_{11}+W_{12}(1-p)$ is the mean fitness of individuals that carry allele $\mathrm{A}_{1}$. Equations (b) and (d) describe coupled population and genetic dynamics.

Now consider the fate of the fitter allele when it is rare and the immigrant type is at demographic equilibrium. To a good approximation, $N \approx N^{\prime}, \bar{W}_{1} \approx W_{12}$, and Equation (d) reduces to $p^{\prime} \approx p W_{12}$. Hence, allele $\mathrm{A}_{1}$ increases in frequency if and only if $W_{12}>1$. In other words, as in the simpler model described in the text, the initial spread of a locally favored allele depends upon its absolute - not relative fitness. Moreover, the alleles that can deterministically increase when rare, can also permit the local population to persist without immigration. 


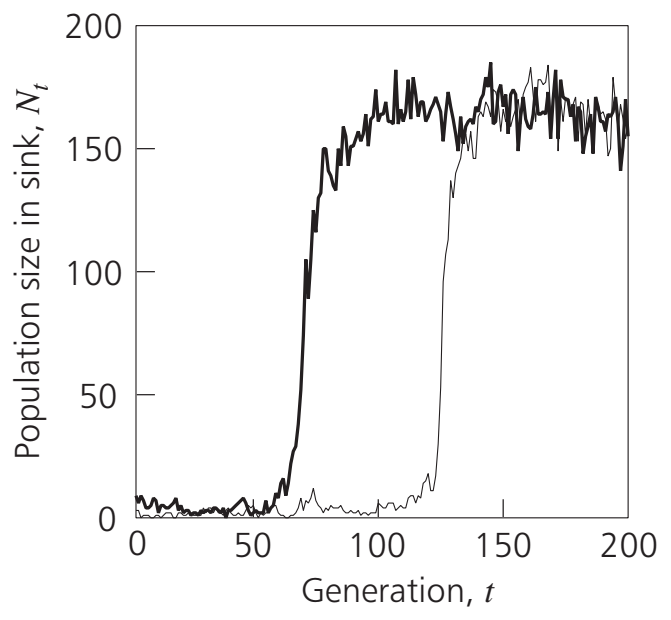

Figure 13.4 Examples of population dynamics in sink populations undergoing adaptive evolution. The abundances shown are measures after selection and before density regulation. Parameters of the individual-based model (see Box 13.2) are the same as in Figure 13.3a with $K=64$, but there is now recurrent immigration. Two characteristic runs are shown, for identical initial populations, with constant immigration rates of $I=4$ adults per generation. The optimum phenotype in the sink is $\hat{z}_{1}=2.8$; the mean phenotype of immigrants from the source is $\hat{z}_{2}=0$. Typically, the sink population stays at low abundance for a lengthy period of time, followed by a period of rapid increases to high abundance, which corresponds to a rapid shift in mean phenotype (see Figure 13.5 for two snapshots of this evolutionary process).

To counter the negative effect of immigration, given density dependence, is the potential role of immigration as a source of genetic variation for local selection, which may be quantitatively much more important than local mutation. Gomulkiewicz et al. (1999) examined this effect in detail for a stochastic model and concluded that the scope for local adaptation in a sink is often greatest at intermediate levels of immigration.

We complement these simple analytical one-locus models of adaptive evolution in sinks with individual-based simulation studies of multilocus evolution, using the model introduced in Box 13.2 to describe ongoing evolution in coupled sources and sinks (Holt et al., unpublished). A large number of source-sink population pairs are tracked, in each of which a fixed number of immigrants per generation is drawn from a stable source population at its mutation-selection-drift equilibrium. Figure 13.4 shows two typical simulation runs. In these examples, the sink environment is harsh, so immigration maintains a population at low abundance only. A population stays in this state for a while (often a long while), but then increases in mean fitness and rapidly grows until limited at the local carrying capacity. Examination of the character states shows that evolution in this system is, in effect, "punctuational": the sink population is either maladapted or near the local optimum, and spends very little time between these two (Figure 13.5), unless immigration is very large relative to the local carrying capacity. Indeed, in these simulations, if immigration is cut off once a population is adapted, the population 


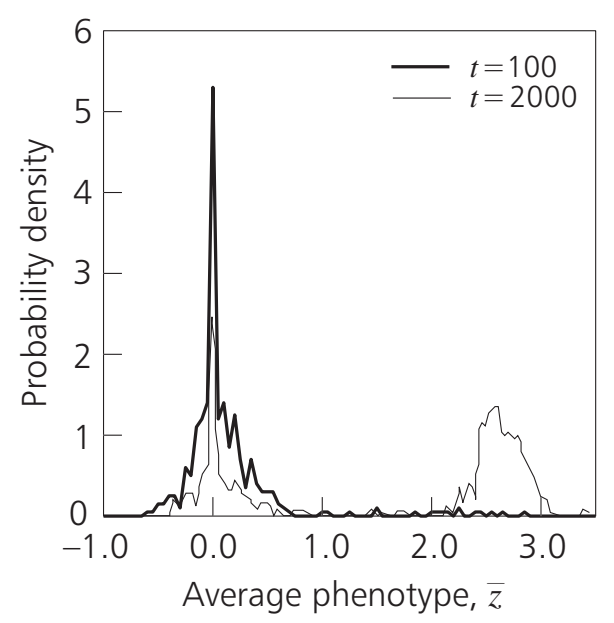

Figure 13.5 Punctuational evolution in a sink habitat. The distribution of average phenotypes $\bar{z}$ in 400 populations is shown at two different times. After 100 generations, most populations are still near the source phenotype $\hat{z}_{2}=0$. After 2000 generations, a substantial number of populations are near the sink optimum $\hat{z}_{1}=3$ (notice that mean phenotypes tend to be somewhat displaced from 3 because of recurrent gene flow from the source). Very few populations are in an intermediate state of adaptation. Unless migration is very high (relative to the local carrying capacity), populations in the sink can thus be dichotomized into being in a "maladapted" state (near the source optimum), or in an "adapted" state (near the sink optimum). Consequently, in a given generation sets of equivalent populations can be characterized by a "probability of adaptation". Other parameters are as in Figure 13.4.

continues to persist for a very long time. This near-dichotomy in degree of adaptation provides a convenient diagnostic with which to summarize large numbers of simulation runs by determining the probability that a population is "adapted" (with a mean genotype near the local optimum, and the population close to its carrying capacity), or "maladapted" (with a mean genotype near that of the source habitat, and a much lower population size), with few populations at intermediate levels of adaptation.

Figure 13.6 shows a typical example of the probability of adaptation that occurs over 1000 generations as a function of the magnitude of initial maladaptation (a measure of the difference between the source and sink environments), and at two different immigration rates. A species that does not adapt over this time scale exhibits niche conservatism. There are several things to note in Figure 13.6:

- We compare Figure 13.6 with Figure 13.3a (for $K=32$ ). In Figure 13.3a, most closed populations exposed to a degree of maladaptation of 2.5 go extinct. By contrast, if these populations were open, drawing immigrants each generation from a source habitat, in each case they would eventually adapt. This illustrates a simple, but fundamental, role of immigration in heterogeneous environments - immigration sustains populations and thus provides an opportunity for evolution. Immigration, in essence, facilitates adaptation to the local environment by the repeated exposure of individuals to it. 


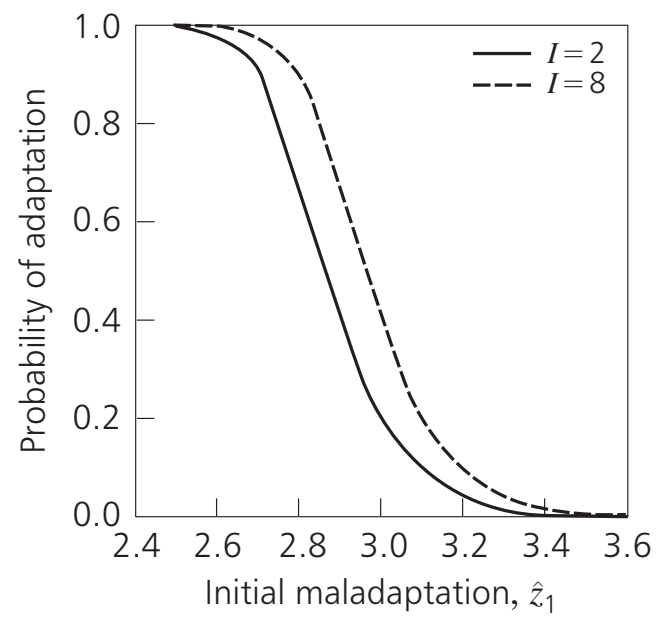

Figure 13.6 The probability of adaptation to the sink environment (within 1000 generations) as a function of sink maladaptation $\hat{z}_{1}$ relative to the source for two different immigration rates, $I=2$ and 8 . Whereas adaptation becomes more difficult the more different the sink environment is from the source environment, adaptation is facilitated by increased immigration. Results shown are averages over 400 populations. Other parameters are as in Figure 13.3a with $K=32$.

- Note that the harsher the sink environment is (as measured by the maladaptation of immigrants), the less likely is adaptive evolution. This corresponds with the other theoretical results sketched above.

- Figure 13.6 shows that the probability of adaptation actually increases with an increasing number of immigrants per generation. Rather than gene flow swamping selection, in the initial phases of adaptation to a novel environment immigration facilitates adaptation. The reason for this is quite simple. Evolution requires variation. A sink population tends to be low in numbers and thus is not likely to retain variation, or generate much variation by mutation. The main source of variation in a low-density sink population is immigration from more abundant persistent sources. Increasing the immigration rate in effect increases the sample of variation drawn each generation from the source. Thus, a higher rate of immigration fuels adaptation by providing more raw material for selection to act upon in the local environment. [See Gomulkiewicz et al. (1999) for a detailed exploration of this effect in the one-locus model of Box 13.3.]

- Once a population has adapted and increased to the carrying capacity, the mean phenotype is displaced from the local optimum (see Figure 13.5, in which the mean genotype of adapted populations is lower than the local optimum of 3). Immigration has two distinct negative effects that hamper local adaptation. First, gene flow from the source (in which there is a different phenotypic optimum) introduces individuals with locally suboptimal phenotypes, who mate with better-adapted residents. This gene flow hampers the perfection of local adaptation. Moreover, at the carrying capacity immigrants compete with residents. This tends to lower absolute fitness, and thereby makes it 
harder for locally superior mutants to spread in the local population (Holt and Gomulkiewicz 1997a, 1997b; Gomulkiewicz et al. 1999).

These recent theoretical results suggest a bias in the diverse roles immigration plays in local adaptation. Immigration facilitates adaptive evolution by exposing species to novel conditions, and also by providing a potent source of genetic variation. Immigration also constrains adaptive evolution, because gene flow can swamp locally favored variants, and because immigrants can compete with betteradapted residents. In the initial stages of adaptation to harsh sink habitats, however, the former effects seem to outweigh the latter.

The models discussed here all involve "black-hole" sink habitats, with unidirectional flows out of source habitats. Studies of comparable one-locus and individual-based models with reciprocal back-flows lead to results broadly consistent with the findings summarized here (Kawecki 1995, 2000; Holt 1996; Ronce and Kirkpatrick 2001; Kawecki and Holt 2002). In particular, the insight that the worse the sink environment, the less likely that adaptive evolution will occur there (over some defined time period) appears quite robust.

\subsection{Adaptations along Environmental Gradients}

So far we have focused on evolution in spatially discrete environments. Such settings offer a useful starting point, but it is important to consider a broader range of spatial scenarios. Another useful limiting case is to imagine that a species is distributed along a smooth environmental gradient that influences both population dynamics and adaptive evolution. Here, we provide a brief overview of work by other authors who have taken this approach, and relate their findings to the discrete-environment models presented in the preceding section.

In a seminal study, Pease et al. (1989) developed a model for a population that grows, adapts, and disperses along a unidirectionally shifting environmental gradient. The model splices a submodel for local population dynamics (exponential growth or decline) with a submodel for local adaptive evolution (for a single quantitative trait that affects fitness). Dispersal influences both the dynamics of abundances (in which individuals on average move from high-density to lowdensity sites) and the character evolution (in which such movements displace local populations away from their local phenotypic optima). The model is described in Box 13.4. It leads to several predictions, which broadly match the results described above for evolutionary rescue in abruptly changed environments (Figure 13.2):

- A species is more likely to persist when the environment changes slowly than when the environment changes rapidly;

- The scope for persistence is enhanced with greater genetic variation, and with a greater maximal growth rate.

The influence of movement upon persistence is more complex. Without dispersal, the model by Pease et al. (1989) predicts that local populations are doomed in a constantly changing environment, even if genetic variation is abundant. Persistence thus requires movement, so a species can track suitable habitats. However, 
movement also tends to move individuals from productive zones along the gradient into unproductive habitats. If sufficiently large, this reproductive drain can cause extinction. This implies an "optimal" rate of movement, measured by the maximal environmental change a species can tolerate. This optimal rate increases with the amount of genetic variation available for selection. The reason is that this increases the relative importance of local selection versus gene flow in determining local phenotypes, which increases the range of environments over which a species will be reasonably well adapted.

Important studies by Kirkpatrick and Barton (1997) and Case and Taper (2000) explore the interplay of gene flow and selection along fixed environmental gradients. Box 13.4 also contains a sketch of the Kirkpatrick-Barton model, which adds density dependence to the Pease et al. (1989) formulation. Also, in the Kirkpatrick-Barton model a species occupies a gradient, along which the optimal value for a phenotypic character changes. The mean phenotype of a population influences its growth rate, and hence its realized density; maladaptation depresses local population size. Gene flow from central populations can inhibit adaptation at the periphery, which depresses fitness and thus local population size; peripheral populations therefore tend to be demographic sinks, maladapted to their environment. This model predicts that if the gradient is sufficiently steep, a species' range can be sharply limited by gene flow. Indeed, a species may not be able to persist at all. By contrast, if the gradient is shallow, gene flow does not prevent local adaptation, and a species' range can expand to fill all available space.

There is an interesting implication of the Kirkpatrick-Barton model for conservation. Assume a species is limited in its range along a gentle gradient by gene flow and swamps local selection at the range margins. If human activity now further sharpens the abundance gradient, the swamping effect of gene flow is magnified relative to local selection. This leads to a degradation in local adaptation, and an evolutionary reduction in the range, as marginal populations become yet more maladapted to their local environments. This is an example of evolution hampering conservation goals.

There are similarities, and differences, between the predictions of the Kirkpatrick-Barton continuum model and those of the discrete-space source-sink models discussed above. In the discrete-sink model, the worse a sink environment is, the harder it is for local adaptation to occur. Likewise, in the continuum model, a sharper gradient implies larger differences in locally optimal phenotypes, so dispersers from sources experience lower fitness in the peripheral sinks. The sharper the gradient, the larger is this drop in fitness for immigrants, and the more likely it is that gene flow prevents local adaptation and thus constrains the range.

However, the models do differ in the predicted role of immigration. In the Kirkpatrick-Barton model, increased immigration can depress population size and even cause extinction (because too many individuals are drained from sources into peripheral sink habitats to which they are maladapted). In the discrete source-sink models, immigration could inhibit local adaptation for ecological reasons (given strong density dependence in the sink), but it can also facilitate local adaptation 
Box 13.4 Modeling adaptation along smooth environmental gradients

Here we describe models used to analyze adaptation and range shifts of populations that live in habitats with smooth environmental gradients. We explain a model by Pease et al. (1989) before introducing its extension by Kirkpatrick and Barton (1997).

The Pease et al. (1989) model has three components. First, changes in population size $N$ at position $x$ along a gradient are modeled by the reaction-diffusion equation

$$
\frac{\partial N}{\partial t}=\frac{1}{2} \sigma^{2} \frac{\partial^{2} N}{\partial x^{2}}+N r
$$

The first term assumes individuals move at random over short distances ( $\sigma$ is the root-mean-square distance moved per time unit), whereas the second term describes local population growth at a per capita rate $r$ that can depend on population size $N$ and on the mean phenotype $\bar{z}$ of individuals at the considered location $x$. Evolution occurs at a single, quantitative character, of mean phenotype $\bar{z}$, influenced by many loci, each of small effect. The local evolutionary dynamics of $\bar{z}$ incorporate gene flow and selection

$$
\frac{\partial \bar{z}}{\partial t}=\frac{1}{2} \sigma^{2} \frac{\partial^{2} \bar{z}}{\partial x^{2}}+\sigma^{2} \frac{\partial \ln N}{\partial x} \frac{\partial \bar{z}}{\partial x}+V_{G} \frac{\partial r}{\partial \bar{z}} .
$$

The first two terms describe how movement modifies the mean character value at a location along the gradient. The third term describes the response of the population to selection, which depends both upon genetic variability $V_{G}$ and on the strength of the relationship between the character and fitness.

The final model component is the expression that describes fitness, which links the two above equations. Pease et al. (1989) were concerned with global extinction versus persistence, so they assumed density-independent growth described by the bivariate function of spatial position and mean phenotype

$$
r=r_{0}-\frac{(x-v t)^{2}}{2 W_{11}\left(1-\rho^{2}\right)}+\frac{\rho \bar{z}(x-v t)}{\left(1-\rho^{2}\right) \sqrt{W_{11} W_{22}}}-\frac{\bar{z}^{2}}{2 W_{22}\left(1-\rho^{2}\right)} .
$$

This function describes how fitness depends jointly upon spatial position (in a timedependent way), and deviations in mean phenotypes from local optima. The parameters describe how wide (or fat) the fitness function is along two dimensions, one being the phenotypic dimension (for $W_{22}$ ), and the other the spatial dimension $x-v t$ (for $W_{11}$ ). The maximal per capita growth rate is achieved only in a population at spatial position $v t$, given that the mean phenotype there is 0 ; $v$ is the velocity of movement of the gradient. The magnitude of spatial variation in the phenotype optimum is given by $\rho$, the correlation between location and the value of the optimal character. The second term measures how fitness decays in space away from the (current) spatial optimum. The final term measures how character variation away from a local optimum translates into reduced fitness.

With these expressions at hand, and assuming that selection is weak, Pease et al. (1989) showed that the maximal rate of environmental change a species can withstand is

continued 
Box 13.4 continued

$$
v_{\max } \approx \sigma \sqrt{2 r_{0}+\frac{V_{G} \rho^{2}}{W_{22}\left(1-\rho^{2}\right)}-\sqrt{\frac{\sigma^{2}}{W_{11}\left(1-\rho^{2}\right)}}} .
$$

Inspection of this equation leads to the conclusions stated in the text.

Kirkpatrick and Barton (1997) use the same dynamic equations, but assume that local growth is density dependent and given by

$$
r=r_{0}(1-N / K)-\frac{1}{2 \omega}[\hat{z}(x)-\bar{z}]^{2}-\frac{I_{S}}{2} .
$$

The first term describes logistic population growth, the second term defines how population growth is depressed if the local mean phenotype $\bar{P}$ deviates from the local optimum $\hat{z}(x)$, with $\omega$ being an inverse measure of the strength of stabilizing selection, and the third term measures the intensity of selection in units of phenotypic variance $V_{P}, I_{S}=V_{P} / \omega$. The optimum is assumed to change linearly with space, $\hat{z}(x)=g x$, in which the quantity $g$ determines the steepness of the environmental gradient.

Kirkpatrick and Barton (1997) considered a number of limiting cases of their model. When dispersal is high, and the population is well below carrying capacity, the maximal gradient slope that permits persistence is approximately $g=$ $\sqrt{V_{P}}\left[4 r_{0}-\left(2-h^{2}\right) I_{S}\right] /\left(2 \sigma \sqrt{I_{S}}\right)$. This expression implies that persistence is facilitated if:

- The environmental gradient is shallow;

- Dispersal is low;

Maximal growth rate is high;

- Selection is weak;

Heritability is high.

by providing novel genetic variation on which selection can act. The latter effect is not dealt with in the continuum model, which assumes that heritability is fixed. Other differences between the models should be considered in future studies. The continuum model assumes homogeneous bidirectional dispersal, so source populations can be depressed by a net loss of emigrants into sinks. The rates of emigration examined in the discrete source-sink models discussed above were low enough for this effect to be ignored. An open challenge for future work is to develop models for evolution (along gradients comparable to those of the Kirkpatrick-Barton model) that also include the positive effect of migration as a source of novel genetic variation in peripheral populations.

Case and Taper (2000) recently combined the Kirkpatrick-Barton model with a Lotka-Volterra model of interspecific competition to examine the interplay of character displacement and range limitation along a gradient. Space limitations here preclude a full discussion of their results, but it is worth noting that, in effect, an interspecific competitor at one end of a gradient sharpens the gradient, which 
makes it more likely that gene flow can limit range size. In the source-sink models, the reason a given habitat becomes a sink may well be the presence of effective competitors or predators. Changes in community structure can thus lead to additional changes via evolutionary responses (see also Chapters 16 and 17). This is an important and largely unexplored dimension in evolutionary conservation biology.

\subsection{Conservation Implications}

There are several general conservation implications that emerge from this wide range of models. A robust result in all the models, involving changes in both time and space, is that populations exposed to "mild" environmental degradation may often be rescued from extinction by evolution. By contrast, severe changes are either likely to lead to global extinction or to the persistent restriction of a species to remnant habitats in which it is already well adapted, without adaptation to novel environments.

The specific models have additional implications. For the model of evolutionary rescue in an abruptly changing environment, the basic message for our rapidly changing world, alas, is sobering. If the environment changes sufficiently fast so that a species in its initial state reaches low densities over short time scales (e.g., tens of generations), natural selection will be rather ineffective at preventing extinction. However, the theory does suggest two distinct avenues through which we might conceivably influence evolution so as to foster conservation:

- Since population size is the product of density and area, populations in large areas take longer to decline to a given absolute abundance than do populations in small areas. This justifies the conservation of large fragments (beyond the usual reasons). Habitat fragments maintained as reserves are likely to continue to experience a broad range of secular changes in the environment, and species in large fragments, in effect, enjoy a "demographic buffer" against unanticipated future environmental changes that may require evolutionary responses by a species for it to persist.

- If the rate of decline can be slowed, populations have an enhanced "window of opportunity" in which to evolve adaptations to environmental stresses. So, if we cannot prevent environmental change, we may be able to reduce the magnitude of its impact upon a focal species. This lengthens the time scale available for evolutionary change and provides more opportunity for evolution by natural selection to alter the species' niche sufficiently to ensure persistence in the novel environment.

The models considered in this chapter highlight that we should not automatically assume that if a habitat is a demographic sink for a species, that habitat has no conservation value. Such habitats may provide sites within which adaptive evolution by the species could occur, and so facilitate its ultimate persistence over a wider landscape than that provided by the current source habitats alone. A conservationist faced with a choice of habitats should obviously attempt to save source 
habitats first. Without these, the species as a whole is doomed over short ecological time scales. When possible, it is also clear that the conversion of current source habitats into future sink habitats should be prevented. However, if such conversion has already occurred, and the landscape is static, it may still be useful to attempt to save or improve sink habitats, particularly if the sinks are "mild" sinks in which substantial populations can be sustained by a trickle of immigration from source habitats. According to the source-sink models discussed here, these are the sites within which significant adaptive evolution to novel environments may occur, buffering the species against further changes in the landscape that destroy or degrade its original required habitat. In the dynamic landscape of the Pease et al. (1989) model, the crucial leading edge of a species' range comprises sink populations that provide a toe-hold for a species to shift its range and track changing environmental conditions.

The source-sink models suggest a management strategy that may sometimes be feasible. What matters in adaptive evolution is the overall demographic contribution of a habitat, and the pattern of coupling by movement among habitats. We may indirectly be able to facilitate local adaptation to, say, a novel toxin encountered in a given habitat by improving resources or other habitat conditions, or by removing a predator. These environmental modifications increase the overall fitness and provide a demographic "boost", which in turn facilitates the efficacy of selection on traits that reduce the fitness impact of the toxin.

The range of models reviewed here reveals that dispersal has disparate effects upon species' survival. In continually changing environments, dispersal may be essential for persistence, but too much dispersal can lead to a substantial mortality load because individuals move into habitats to which they are maladapted. The latter effect can constrain species' ranges in constant environments, and may even threaten persistence when environmental gradients are steep. To counter the latter negative effect of dispersal, genetic variation can be increased and so the capacity of a species to respond to change is enhanced (see Figure 13.3c). The ultimate source of all variation is, of course, mutation, but in many local populations mutational input may be minor relative to another source of variation - gene flow from spatially separated populations. This effect has just begun to be explored, and the results provided above suggest that in some circumstances it could be an important avenue through which immigration facilitates adaptive evolution. This version of the effects of dispersal upon evolution and persistence complicates analyses of the ultimate conservation importance of different patterns of landscape connectivity and warrants further investigation.

\subsection{Concluding Comments}

Conservation problems exist because humans change the environment in ways that harm species (as measured in distribution and abundance), because species do not adapt by natural selection to these novel environments, and because actual adaptations eventually harm populations. The theoretical studies sketched above suggest 
that, in some situations, the evolutionary dynamics of populations may be harnessed to facilitate species' preservation. However, these same theoretical results suggest that evolution will not be particularly useful in promoting the persistence of species in radically changed environments, or in environments that comprise a spatial admixture of unchanged and greatly altered habitats. The basic message is that the demographic context of evolution matters greatly in determining the likelihood of conservatism versus rapid evolution in altered environments, and that this insight should be useful in applied conservation biology. In particular, we have seen that in spatially heterogeneous environments evolution by natural selection improves adaptation less effectively in local environments in which fitness and population size are both low (as, for example, in a sink habitat, or near the margin of a species' range). The sharper the difference in fitness between source and sink, or the steeper the gradient, the more likely is an absence of evolutionary response to spatial heterogeneity. Conversely, adaptive evolution is likely along gentle environmental gradients.

Acknowledgments We thank the National Science Foundation for its support (DEB9528602). We particularly thank Michael Barfield, who has been a collaborator on much of the work summarized here, and assisted in figure preparation. 


\section{References}

References in the book in which this chapter is published are integrated in a single list, which appears on pp. 365-410. For the purpose of this reprint, references cited in the chapter have been assembled below.

Bradshaw AD (1991). Genostasis and the limits to evolution. Philosophical Transactions of the Royal Society of London B 333:289-305

Bürger R \& Lynch M (1995). Evolution and extinction in a changing environment: A quantitative genetic analysis. Evolution 49:151-163

Burt A (1995). Perspective: The evolution of fitness. Evolution 49:1-8

Case TJ \& Taper ML (2000). Interspecific competition, environmental gradients, gene flow, and the coevolution of species' borders. The American Naturalist 155:583-605

Falconer DS (1989). Introduction to Quantitative Genetics, Third Edition. New York, NY, USA: John Wiley and Sons

Fisher RA (1958). The Genetical Theory of Natural Selection, Second Revised Edition. New York, NY, USA: Dover Publications

Gomulkiewicz R \& Holt RD (1995). When does evolution by natural selection prevent extinction? Evolution 49:201-207

Gomulkiewicz R, Holt RD \& Barfield M (1999). The effects of density dependence and immigration on local adaptation and niche evolution in a black-hole sink environment. Theoretical Population Biology 55:283-296

Haldane JBS (1930). A mathematical theory of natural and artificial selection. VI. Isolation. Proceedings of the Cambridge Philosophical Society 26:220-230

Hedrick PW (2000). Genetics of Populations, Second Edition. Sudbury, MA, USA: Jones and Bartlett

Holt RD (1990). The microevolutionary consequences of climate change. Trends in Ecology and Evolution 5:311-315

Holt RD (1996). Demographic constraints in evolution: Towards unifying the evolutionary theories of senescence and niche conservatism. Evolutionary Ecology 10:1-11

Holt RD \& Gaines MS (1992). The analysis of adaptation in heterogeneous landscapes: Implications for the evolution of fundamental niches. Evolutionary Ecology 6:433-447

Holt RD \& Gomulkiewicz R (1997a). How does immigration influence local adaptation? A re-examination of a familiar paradigm. The American Naturalist 149:563-572

Holt RD \& Gomulkiewicz R (1997b). The evolution of species' niches: A population dynamic perspective. In Case Studies in Mathematical Biology, eds. Othmar HG, Adler FR, Lewis MA \& Dallon JC, pp. 25-50. Englewood Cliffs, NJ, USA: Prentice-Hall

Holt RD, Gomulkiewicz R \& Barfield M. The phenomenology of niche evolution via quantitative traits in a "black-hole" sink: A mechanism for punctuated evolution? Unpublished

Kawecki TJ (1995). Demography of source-sink populations and the evolution of ecological niches. Evolutionary Ecology 7:155-174

Kawecki TJ (2000). Adaptation to marginal habitats: Contrasting influence of dispersal on the fate of rare alleles with small and large effects. Proceedings of the Royal Society of London B 267:1315-1320

Kawecki TJ \& Holt RD (2002). Evolutionary consequences of asymmetric dispersal rates. The American Naturalist 160:333-347

Kirkpatrick M \& Barton NH (1997). Evolution of a species' range. The American Naturalist 150: $1-23$

Lynch M, Conery J \& Bürger R (1995a). Mutation accumulation and the extinction of small populations. The American Naturalist 146:489-918 
Orr HA (1998). The population genetics of adaptation: The distribution of factors fixed during adaptive evolution. Evolution 52:935-949

Pease CM, Lande R \& Bull JJ (1989). A model of population growth, dispersal, and evolution in a changing environment. Ecology 70:1657-1664

Pulliam HR (1996). Sources and sinks: Empirical evidence and population consequences. In Population Dynamics in Space and Time, eds. Rhodes OE, Chesser RK \& Smith MH, pp. 45-70. Chicago, IL, USA: University of Chicago Press

Ronce O \& Kirkpatrick M (2001). When sources become sinks: Migrational meltdown in heterogeneous habitats. Evolution 55:1520-1531

Schoener TW (1989). The ecological niche. In Ecological Concepts, ed. Cherrett JM, pp. 79-114. Oxford, UK: Blackwell

Watkinson AR \& Sutherland WJ (1995). Sources, sinks and pseudo-sinks. Journal of Animal Ecology 64:126-130 